\title{
Altering sub-cellular location for bioimaging by engineering the carbon based fluorescent nanoprobe
}

\author{
Chenchen $\mathrm{Li}^{1,2 \dagger}$, Kangkang Zhang ${ }^{1 \dagger}$, Chenjie Yao ${ }^{1,4}$, Lin Ding ${ }^{1,5}$, Yanan Huang ${ }^{1,2}$, Xuelian Yin ${ }^{1,2}$, \\ Junfeng Zhang ${ }^{1,2}$, Wenchao Gao ${ }^{1,2}$, Jian Zhang ${ }^{3}$, Minghong $\mathrm{Wu}^{1^{*}}$ and Yanli Wang ${ }^{1,2^{*}}$
}

In recent years, numerous classes of carbon-based nanomaterials, such as carbon nanotubes (CNTs), carbon dots (CDs), graphene and its derivatives, graphene quantum dots (GQDs) and fullerene, have been deeply explored for potential applications in the biological fields, e.g., bioimaging [1-5], biosensing [6,7], drug nanocarrier [8-12], etc., owing to their unique and alluring physical and chemical properties. Among them, GQDs are a subject of interesting and promising research with many advantages such as strong signal strength, resistance to photobleaching, tunable fluorescence emissions, high sensitivity and biocompatibility [13-35]. Compared with those semiconductor QDs, GQDs have remarkable superiority in low toxicity, excellent biocompatibility, low cost, and abundance of original materials in nature [36]. High quality GQDs have a wide range of applications, such as light emitting diodes (LEDs) [37,38], solar cells $[39,40]$ and photocatalysis $[41,42]$, aside from biological fields. Up to now, various GQDs with different photoluminescent (PL) colors have been synthesized by two dominating approaches including top-down and bottomup methods. The top-down method refers to cutting bulk carbon materials into nanoscale-carbon materials by necessary physical and chemical processes [43]. Although this method is feasible to produce GQDs in the laboratory, the tedious and time-consuming experimental procedures limit their large-scale preparation. Furthermore, these GQDs display weak absorption band, and thereby, their fluorescent quantum yields (QY) are usually quite low with low brightness when applied in bioimaging $[44,45]$. The poor optical property is ascribed to the highly defective structures, which are inevitably uncontrollable in the cutting processes from the top-down methods. On the contrary, the bottom-up method involves the formation of GQDs from molecular organic precursors [46], and these routes can effectively obtain controllable morphology and uniform GQDs.

Although several excellent synthetic methods have emerged, some fundamental issues like luminescence mechanism are still controversial. Currently, the luminescence mechanism mainly includes surface/edge state, quantum confinement effect of conjugated $\pi$-domains, and producing fluorescent molecules during the reaction [47]. In fact, the luminescence mechanism of some carbon-based fluorescent nanoprobes may be dominated by the molecular state, in which the luminescence center relies on an organic fluorophore [48]. Some basic phenomena could support this molecular state theory. For example, fluorescent components can quickly penetrate out of the dialysis bag during dialysis [49]. However, the relationship between fluorescence and GQDs self-structure is still not very clear.

Herein, we present a mass production of carbon-based fluorescent nanoprobes with sulfonic groups (S-GQDs), which is built by a facile molecular fusion route under eco-friendly hydrothermal conditions using pyrene $\left(\mathrm{C}_{16} \mathrm{H}_{10}\right)$ as the carbon precursor (Fig. 1a). As reported in our previous work, positively charged amino-GQDs (AGQDs) could easily pass across cell membranes to cell cytoplasm [44]. They have strong fluorescence, but show high toxicity in vitro and in vivo. While negatively charged sulfonic-GQDs, which have been reported as

\footnotetext{
${ }^{1}$ Tumor Precision Targeting Research Center, School of Environmental and Chemical Engineering, Shanghai University, Shanghai 200444, China

${ }^{2}$ Institution of Nanochemistry and Nanobiology, School of Environmental and Chemical Engineering, Shanghai University, Shanghai 200444, China

${ }^{3}$ Universal Medical Imaging Diagnostic Research Center, Shanghai 200233, China

${ }^{4}$ Harvard T.H. Chan School of Public Health, Harvard University, Boston, Massachusetts 02115, USA

${ }^{5}$ Department of Biological Chemistry, The University of Michigan, Ann Arbor, MI 48109, USA

† These authors contributed equally to this work.

* Corresponding authors (emails: mhwu@shu.edu.cn (Wu M); wangyanli@staff.shu.edu.cn (Wang Y))
} 

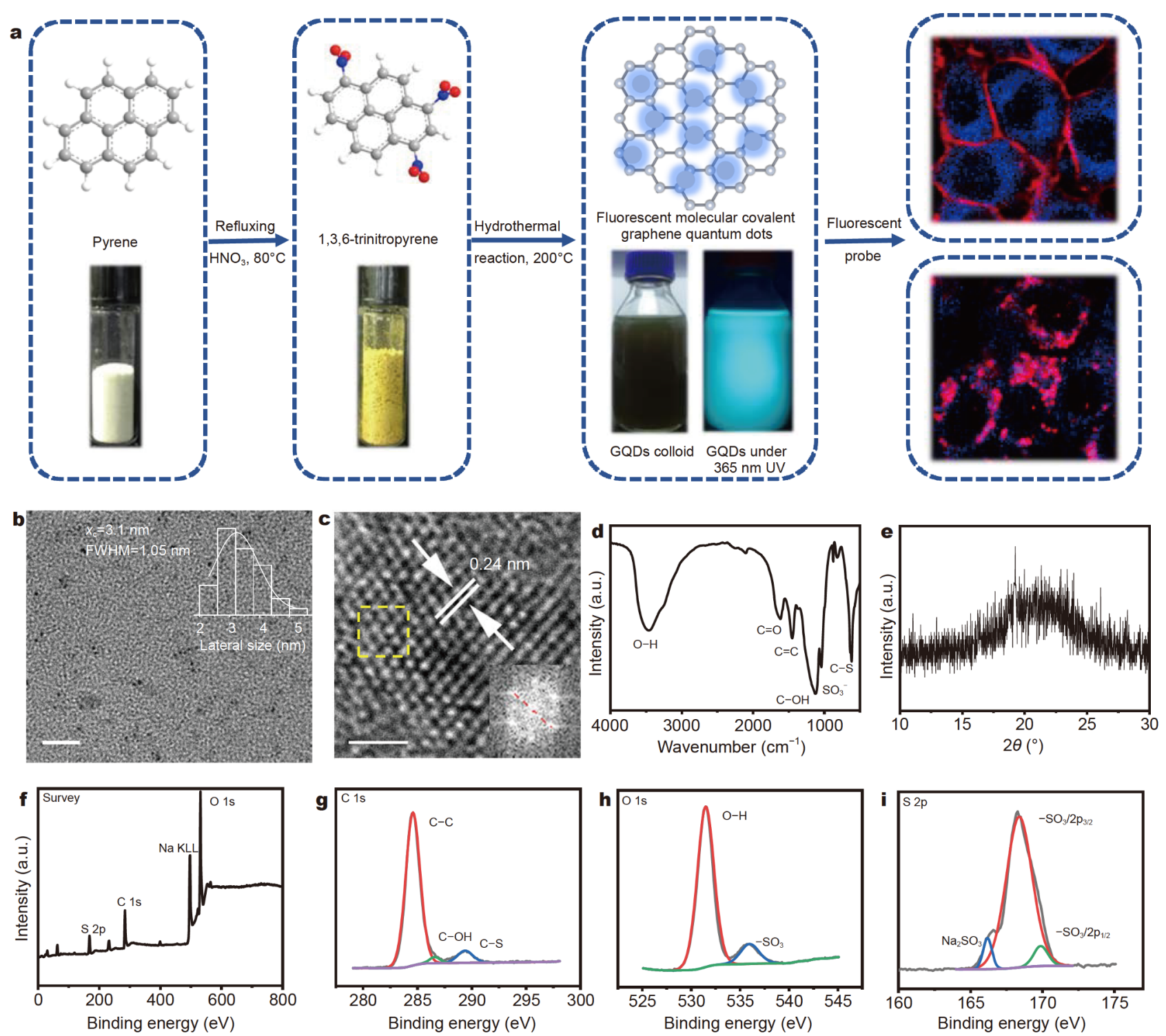

Figure 1 (a) Schematic illustration of the large-scale preparation steps of S-GQDs. (b) TEM image of S-GQDs (size distribution inset). Scale bar: $20 \mathrm{~nm}$. (c) HRTEM image of S-GQDs. Scale bar: $1 \mathrm{~nm}$; the lattice parameter is $0.24 \mathrm{~nm}$. The inset is an FFT image of a corresponding area (selected by the yellow square). (d) The FT-IR spectra of S-GQDs. (e) The XRD pattern of S-GQDs. (f) XPS full survey of S-GQDs. The high resolution XPS of C 1s (g), O 1s (h), and S 2p (i).

graphene-based tumor cell nuclei-targeting fluorescent nanoprobes (GTTNs) [50], cannot pass through the cell membrane even after co-incubation for $48 \mathrm{~h}$ under normal culture conditions. However, the specific capability of GTTN targeting to cancer in vivo and further targeting to cancer cell nuclei was found due to the difference of cell membrane permeabilities between tumor cells and normal cells. Based on this phenomenon, we proposed a new targeting mechanism, named as the cell membrane permeability targeting (CMPT) mechanism [50]. Compared with our previous work, the S-GQDs in this paper are expected to be a new fluorescent probe owing to its low toxicity, transmembrane transport, cellular distribution in lysosome, excellent biocompatibility and high PL QY.

As shown in Fig. 1a, mass production of S-GQDs could be achieved by hydrothermal reaction from the precursor pyrene powder which was transformed to 1,3,6-trinitropyrene by hot $\mathrm{HNO}_{3}$. The obtained 1,3,6-trinitropyrene was re-dissolved in a $\mathrm{NaS}_{2} \mathrm{O}_{3}$ solution and blended with ultrasonic treatment. The mixed suspension 
was transferred to a Teflon-lined autoclave and heated at $200^{\circ} \mathrm{C}$ for $12 \mathrm{~h}$. During the heating, small graphite-like fragments could fuse into larger GQDs structures. After being cooled to room temperature, the resulting black colloidal solution was obtained and purified.

Fig. $1 \mathrm{~b}$ and $\mathrm{c}$ show the transmission electron microscopy (TEM) image and high-resolution TEM (HRTEM) image of individual crystallized S-GQDs. The size distribution of S-GQDs is shown in Fig. 1b (inset), revealing that the size of S-GQDs is mainly distributed in the range of 2-5 $\mathrm{nm}$ with a mean diameter of approximately $3 \mathrm{~nm}$. Besides, Gaussian distribution is suitable for characterizing the size distribution. The full-width at-halfmaximum (FWHM) of the fitted Gaussian curve (Fig. 1b inset) is $1.05 \mathrm{~nm}$, indicating the regular distribution feature of the GQDs [51]. The selected area of HRTEM image by the box has a fast Fourier transform (FFT) image shown in Fig. 1c (inset), demonstrating the distance between the lattice fringes is $0.24 \mathrm{~nm}$, which agrees well with the lattice fringes of graphene [1120] [52,53]. The S-GQDs were functionalized by the chemical groups, whose characteristic signals are shown in FT-IR (Fig. 1d), $\mathrm{C}=\mathrm{O}$ at $1617 \mathrm{~cm}^{-1}, \mathrm{C}=\mathrm{C}$ at $1450 \mathrm{~cm}^{-1}, \mathrm{C}-\mathrm{OH}$ at $1125 \mathrm{~cm}^{-1}, \mathrm{O}-\mathrm{H}$ at $3464 \mathrm{~cm}^{-1},-\mathrm{SO}_{3}$ at $1041 \mathrm{~cm}^{-1}$ and $\mathrm{C}-\mathrm{S}$ at $618 \mathrm{~cm}^{-1}$, indicating that S-GQDs are co-functionalized by $-\mathrm{OH}$ and $-\mathrm{SO}_{3}$ and endowed with relatively good solubility in aqueous solution (AS). XRD analysis (Fig. 1e) conveys the fact of the lattice spacing $(0.39 \mathrm{~nm})$ and diffraction center (about $22^{\circ}$ ) in line with graphite [002]. To study the elemental constituent of the S-GQDs, XPS measurements (Fig. 1f-i), particularly high-resolution $\mathrm{C} 1 \mathrm{~s}, \mathrm{O} 1 \mathrm{~s}$ and $\mathrm{S} 2 \mathrm{~s}$ spectra, were conducted. As shown in Fig. 1f, XPS full survey of S-GQDs indicates the main elements of $\mathrm{C}, \mathrm{O}$, and $\mathrm{S}$ contained in S-GQDs. High-resolution $\mathrm{C}$ 1s spectrum is shown in Fig. 1g, whose peaks are fitted to the main $\mathrm{C}-\mathrm{C}$ feature, $\mathrm{C}-\mathrm{OH}$ and $\mathrm{C}-\mathrm{S}$, which indicates the carbon skeleton of S-GQDs and functional groups. Other notable features originate from the $\mathrm{O}-\mathrm{H}$ bond and $-\mathrm{SO}_{3}$ in Fig. $1 \mathrm{~h}$. The strong signals of $-\mathrm{SO}_{3} / 2 \mathrm{p}_{1 / 2}$ and $-\mathrm{SO}_{3} / 2 \mathrm{p}_{3 / 2}$ (Fig. 1i) are ascribed to the sulfonic-group of S-GQDs.

To detect the optical properties and explore the fluorescent mechanism of S-GQDs, we characterized the UVvis absorption spectrum of S-GQDs in AS and found an absorption band at ca. $240 \mathrm{~nm}$ (Fig. 2a), which was assigned to the $\pi \rightarrow \pi^{*}$ transition of aromatic $\mathrm{sp}^{2}$ domains [54]. Besides, a photograph of the S-GQDs illuminated under UV light $(365 \mathrm{~nm})$ is exhibited. The bright bluegreen fluorescence of GQDs is clearly seen with the naked eye (inset of Fig. 2a). Similar to the GQDs prepared by a hydrothermal graphene oxide reduction method, a typical absorption peak at ca. $290 \mathrm{~nm}$ is observed. Then a near-
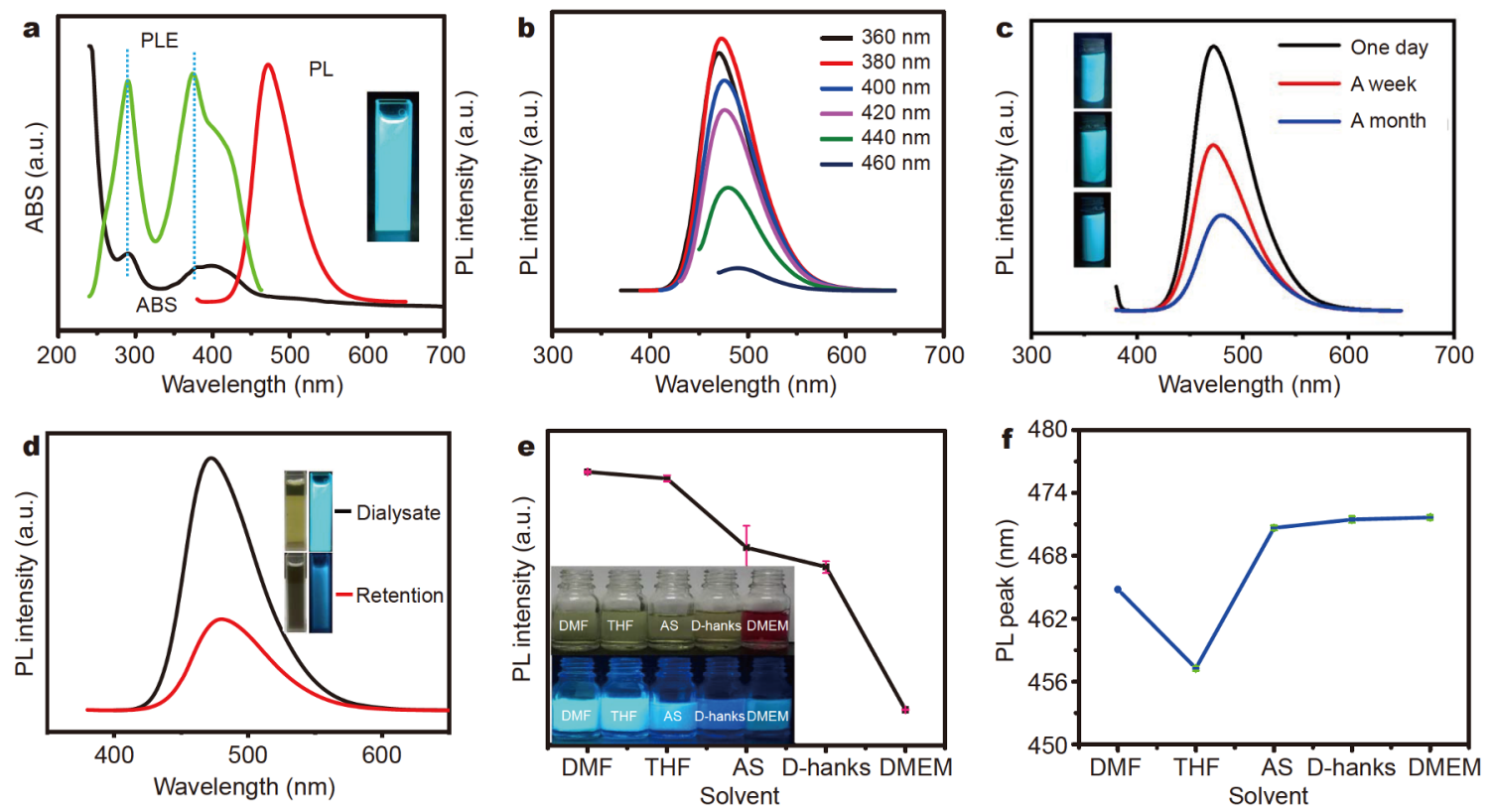

Figure 2 (a) UV-visible absorption (ABS), photoluminescent emission (PL) and photoluminescent excitation (PLE) spectra of S-GQDs. (b) PL spectra of S-GQDs excited at different excitation wavelengths varying from 360 to $460 \mathrm{~nm}$. (c) Photostability of S-GQDs over a long period of time from one day to a month. (d) PL spectra of dialysate and remaining solution after S-GQDs dialysis. (e) Solvent-dependent PL intensity of S-GQDs collected at $373 \mathrm{~nm}$ excitation. (f) Solvent-dependent PL peaks of S-GQDs collected at $373 \mathrm{~nm}$ excitation. 
visible light absorption at about $398 \mathrm{~nm}$ also appears. Both of them can be ascribed to the fluorescent molecules existing at edges or in the carbon core, which trigger strong fluorescence of the obtained S-GQDs [48], and the relative PL QY of the S-GQDs is about 22\% (Fig. S1a). To further explore the optical properties of the as-prepared S-GQDs, a detailed PL study was carried out with different excitation wavelengths (Fig. 2b). Generally, the excitation wavelength dependence of the emission wavelength and PL intensity change is a common phenomenon observed in carbon-based fluorescent materials, which means PL peaks would shift to longer wavelengths when the excitation wavelength increases and the PL intensity would decrease rapidly simultaneously [55]. However, the as-synthesized S-GQDs exhibit an emission wavelength nearly independent of excitation wavelength (Fig. 2b) in the range of $360-460 \mathrm{~nm}$. The PL maximum is well confined, nearly irrespective of excitation wavelength. The maximum excitation wavelength is $373 \mathrm{~nm}$ and the maximum emission wavelength is $480 \mathrm{~nm}$. The reason for this phenomenon is according to fluorescent substances [43]. The strongest PL maximum appears at $480 \mathrm{~nm}$ upon $373 \mathrm{~nm}$ excitation, which is consistent with their PL excitation (PLE) spectrum with a strong excitation peak at $373 \mathrm{~nm}$ (Fig. 2a). The $398 \mathrm{~nm}$ PLE peak also has a corresponding absorption band in UV-vis. The PLE spectrum clearly demonstrates that the observed luminescence from the GQDs is directly correlated with the two new transitions at 290 and $373 \mathrm{~nm}$ rather than the commonly observed $\pi \rightarrow \pi^{*}$ transition [54]. The PL lifetime of the GQDs is 4.932 ns as shown in Fig. S1b.

The photostability of the GQDs was examined by monitoring the change in the PL intensity under continuous radiation using a $100 \mathrm{~W}$ Xenon lamp (Fig. S1c). After irradiation for $3 \mathrm{~h}$, S-GQDs show a high resistance to photobleaching as observed by the PL intensity and emission brightness of the S-GQDs (inset Fig. S1c), indicating that the S-GQDs are extremely photostable during the relatively short time period. However, the fluorescence would tempestuously reduce when S-GQDs were stored for a long time, which suggests that fluorescent molecules are of significance to the fluorescent mechanism (Fig. 2c). According to the dialysis process, we found that the fluorescent intensity of the dialysate was stronger than that of the remaining solution (Fig. 2d), in accord with the fluorescent molecules mechanism. The $\mathrm{pH}$-dependence of PL was also tested in a wide range of $\mathrm{pH}$ values as shown in Fig. S2a. Under alkaline and neutral conditions, the S-GQDs emitted relatively strong PL. Whereas the PL intensity became rapidly reduced under acidic conditions because the chemical groups of the fluorescent molecules coupled with S-GQDs were protonated. Thus, the hydrophilic groups were severely broken, leading to aggregation of GQDs. Then these GQDs became inactive in PL [54]. It should be mentioned that there were strong fluorescent intensities at $\mathrm{pH} 5-12$, indicating enormous potential application in vivo. All the facts implied that the emission was characterized by the exciton-state of molecules/chemical groups rather than quantum effect [45].

Fig. S2b shows the dependence of PL intensity on the concentration of S-GQDs colloidal solution. Different concentrations were tested with the absorbance at $373 \mathrm{~nm}$. At low concentrations (with absorbance less than 0.2 ), a linear relationship is observed. But with increasing absorbance and concentrations, the slope of PL intensity shows a sharp slowdown [51,56] (intrinsic colour variation of various concentrations and the corresponding fluorescent brightness are displayed in the inset of Fig. S2b). We could foresee that the PL intensity would eventually reach a plateau, because high concentrations of GQDs induce the self-absorption quenching of fluorescence. As shown in Fig. 2e, the PL of GQDs decreases in solvents from dimethylformamide (DMF), tetrahydrofuran (THF), AS, D-hanks (buffer solution) to DMEM (culture medium). The S-GQDs in the solvent with strong polarity owned the strongest PL. Whereas, the peaks of maximum PL exhibit negligible solvent-dependent behaviours (Fig. 2f) [54]. Besides, it is distinctly observed that the fluorescence intensity of S-GQDs decreased in these solvents under UV exposure (Fig. 2e inset), proving that these solvents apparently influence the fluorescence of S-GQDs. It is easily understood that ions or solvent molecules attaching to the edges or carbon cores of GQDs prompt the change of marginal fluorescent molecules or photoluminescence related functional groups rather than the impact of conjugated system. Based on these, we could speculate that fluorescent molecule-conjugated GQDs have a pivotal effect on the photoluminescence mechanism of the obtained S-GQDs. When they were excited within a certain excitation wavelength range, those electron-donating chemical groups on the fluorescent molecule-conjugated GQDs enhanced the radiative recombination of localized electron-hole pairs $[48,54]$.

We also investigated the surface charge of S-GQDs in AS and culture medium by zeta potential measurements. As shown in Fig. S2c, the S-GQDs are negatively charged in AS (average value of $-38 \mathrm{mV}$ and absolute value $>30 \mathrm{mV}$ ) and show a rough trend that the S-GQDs 
become more negatively charged when the $\mathrm{pH}$ value changes from 5 to 12 . Referring to the FT-IR spectra, the negative surface charge of S-GQDs is ascribed to the presence of abundant hydroxyl, carboxyl and sulfonic acid groups. From the view of colloidal theory [56], a zeta potential more than $-30 \mathrm{mV}$ is necessary for the stable dispersion of a general colloidal system. Hence, the S-GQDs should be better dispersed in AS than in culture medium. The complex component in culture medium made the unstable S-GQDs agglomerated because of the interactions between them. To investigate the stability of S-GQDs, we did a simple sedimentation experiment for different times and measured their zeta potentials as shown in Fig. S2d. As a result, the S-GQDs become unstable because of the decreased zeta potential in AS as the settling time increases. However, the zeta potentials in culture medium, which are smaller than in AS, do not show noticeable changes over time due to the interactions between S-GQDs and proteins or other complex components in the medium. In other words, the S-GQDs aggregate rapidly in the culture medium and then remain relatively stable as time goes on.

Endowed with superb optical properties such as high QY, the S-GQDs were considered to serve as promising biofluorescent probes. We discussed the difference of the cellular behaviours among the three GQDs, negative SGQDs, GTTN and positive A-GQDs. Bioimaging experiments were performed by a confocal fluorescence microscope as shown in Fig. 3. Bright blue fluorescence was observed in cellular cytoplasm of S-GQDs and A-
GQDs, while the GTTN only bounded and adsorbed on the cell membrane surface without entering the cells after $48 \mathrm{~h}$ incubation (Fig. 3a). As shown in Fig. S3, both SGQDs and A-GQDs entered the cells through the membrane even within $10 \mathrm{~min}$. It is revealed that engineering the nanoparticles through the synthetic route and raw materials could change the biotoxicity and cellular distribution. Indeed, many nanoparticles have been found to enter cells through endocytosis, a fundamental process that eukaryotic cells engulfs substance via the membrane deformation [57]. Lysosome is an important organelle, which is tiny sacs filled with enzymes, playing vital roles in cellular degradation, autophagy, immunological stress, etc. [58]. Most cell uptake endocytic routes of nanoparticles converge upon the lysosome, which is regarded as the most common intracellular site of nanoparticle sequestration and degradation [59]. In addition to the endo-lysosomal pathway, recent evidence suggested that many nanoparticles were found in lysosome, which may cause in vivo and in vitro toxicity [58,60-65]. We found that both S-GQDs and A-GQDs can enter the cell, and locate in lysosome (Fig. 3b) which can be indicated by Pearson's correlation of lysosome colocation after coincubation with S-GQDs, GTTN and A-GQDs for $48 \mathrm{~h}$ (Fig. S4a). The Pearson's correlation of S-GQDs and lysosome was almost 0.6 after $1 \mathrm{~h}$ incubation and increased as the incubation time prolonged (Fig. S4b). S-GQDs exhibit very good biosecurity (i.e., nontoxicity) even with high dose $\left(300 \mathrm{mg} \mathrm{L}^{-1}\right)$ and long incubation time $(48 \mathrm{~h})$ (Fig. 4a, d) while A-GQDs are toxic to cells when the co-

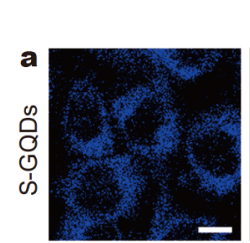

CellMask
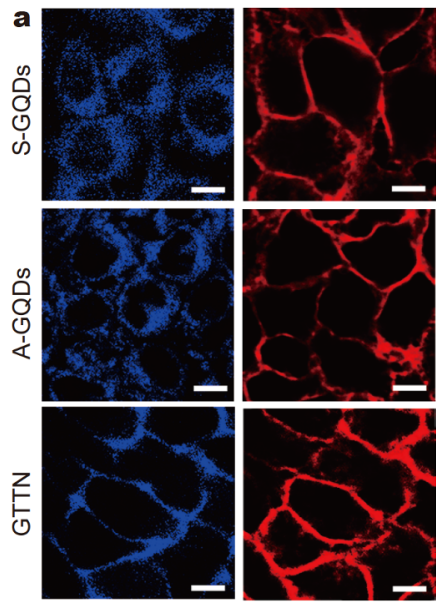

Merge
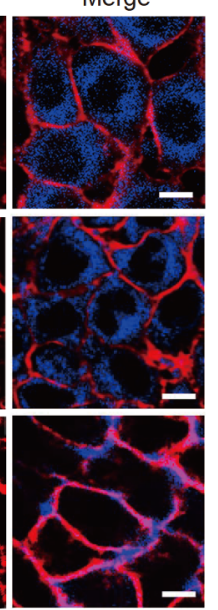

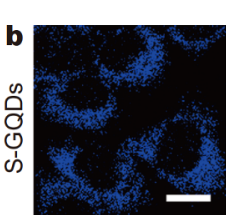

Lysosome
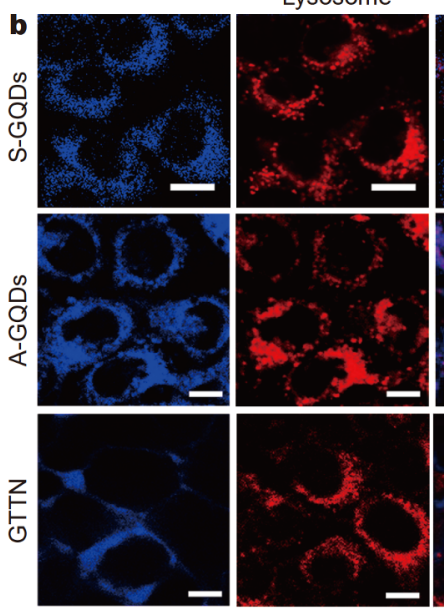

Merge

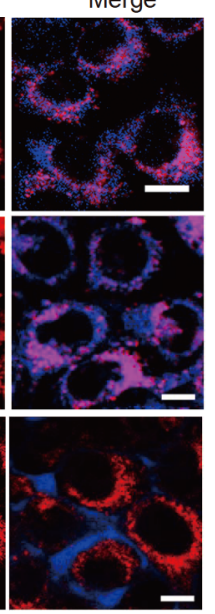

Figure 3 Confocal images of S-GQDs, A-GQDs and GTTN in 4T1 cell in vitro. (a) Cell membrane co-localization. (b) Lysosome co-localization. Cells were co-incubated with $100 \mathrm{mg} \mathrm{L}^{-1}$ S-GQDs, $30 \mathrm{mg} \mathrm{L}^{-1}$ A-GQDs or $100 \mathrm{mg} \mathrm{L}^{-1}$ GTTN for $48 \mathrm{~h}$. Left, graphene-based nanoparticles (405 nm excitation); middle, cell membrane stained by CellMask Orange plasma membrane stain (561 nm excitation) or cell lysosome stained by LysoTracker Deep Red (635 nm excitation); right, merged image. Scale bar: $10 \mu \mathrm{m}$. 

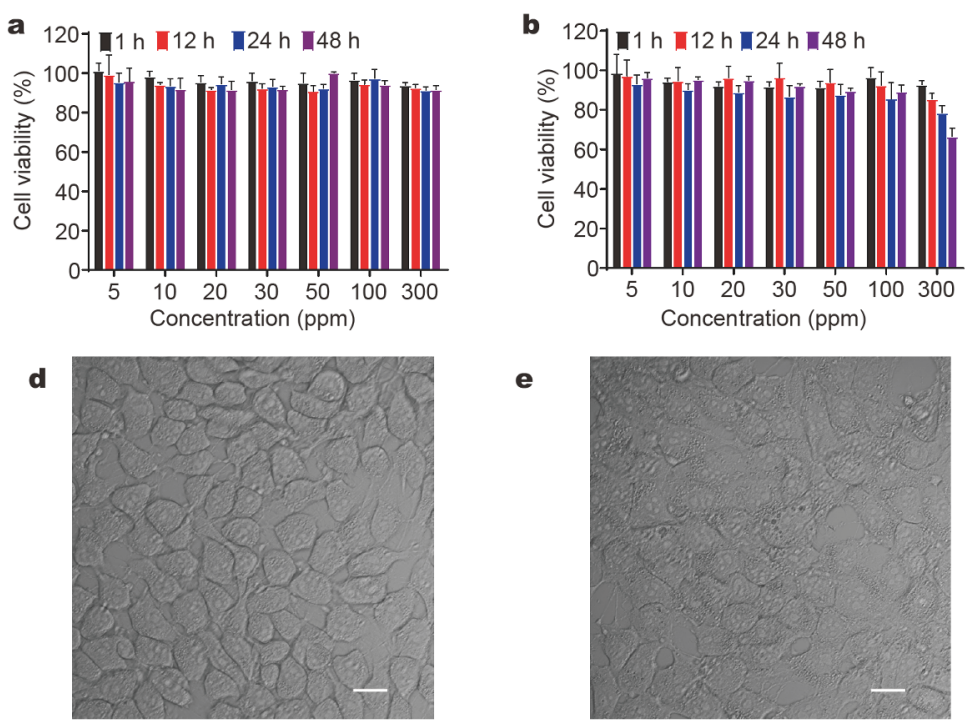

$\mathbf{g}$

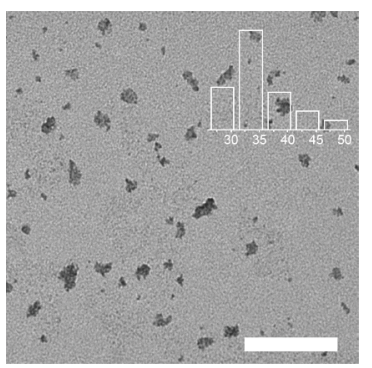

j

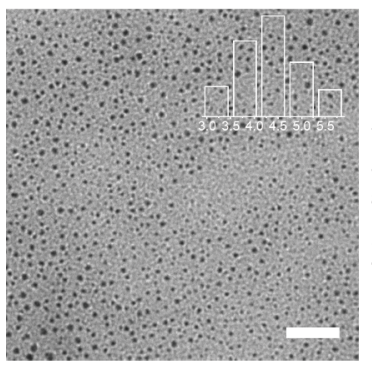

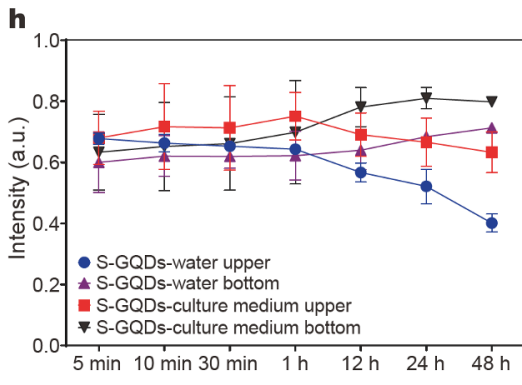

Time

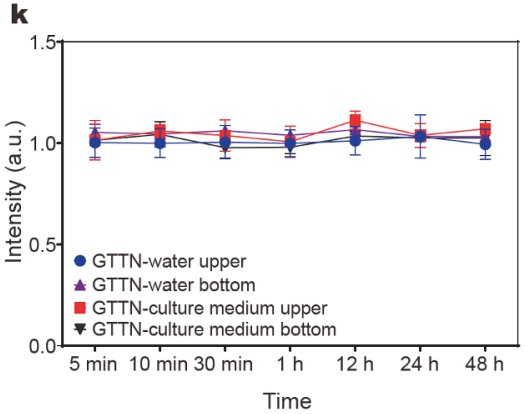

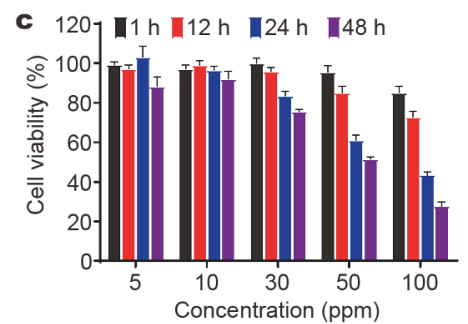

$\mathbf{f}$
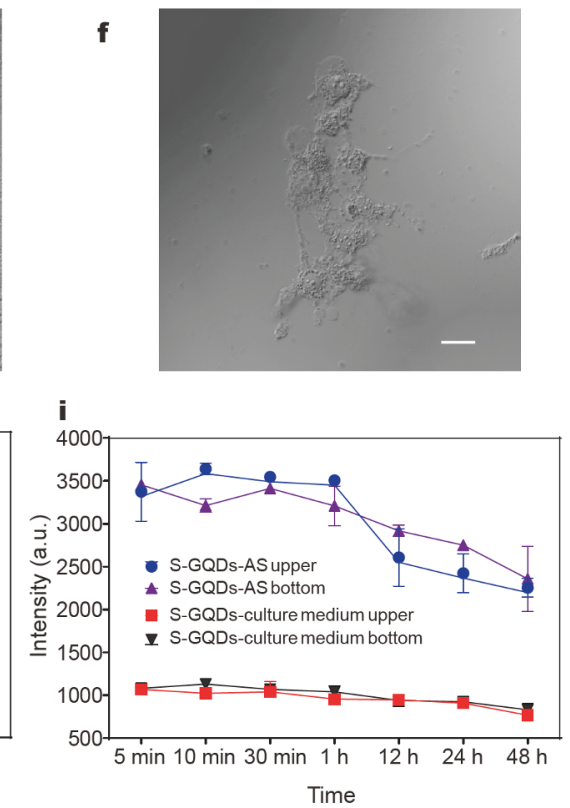

I

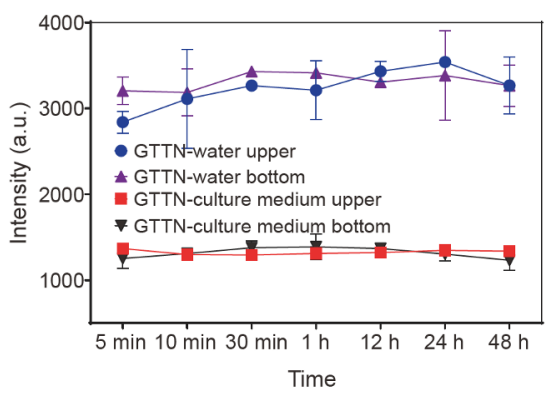

Figure 4 Cell viability assays of (a) S-GQDs, (b) GTTN, and (c) A-GQDs at different concentrations for different incubation times detected by cck-8 assay. Representative light microscopic images of (d) S-GQDs of $300 \mathrm{mg} \mathrm{L}^{-1}$ (48 h, scale bar: $20 \mu \mathrm{m}$ ); (e) GTTN of $300 \mathrm{mg} \mathrm{L}^{-1}$ (48 h, scale bar: $20 \mu \mathrm{m}$ ); (f) A-GQDs of $50 \mathrm{mg} \mathrm{L}^{-1}(48 \mathrm{~h}$, scale bar: $20 \mu \mathrm{m})$. Static settled S-GQDs and GTTN for different times from 5 min to $48 \mathrm{~h}$ in AS and culture medium. TEM images of S-GQDs ( $\mathrm{g}$ ) and GTTN (j) after being settled for $48 \mathrm{~h}$. Scale bars: $200 \mathrm{~nm}(\mathrm{~g}) ; 100 \mathrm{~nm}(\mathrm{j})$. UV-visible absorption peak value changes of S-GQDs (h) and GTTN (k) at $398 \mathrm{~nm}$. Photoluminescent peak value changes of S-GQDs (i) and GTTN (l) at $480 \mathrm{~nm}$ as the settling time increases.

incubation concentration increased to $50 \mathrm{mg} \mathrm{L}^{-1}$ after 24 and $48 \mathrm{~h}$ incubation (Fig. 4c, f). GTTN has low toxicity but still shows adverse impact on cells with the very high dose of $300 \mathrm{mg} \mathrm{L}^{-1}$ after $48 \mathrm{~h}$ incubation (Fig. 4b, e). SGQDs, A-GQDs and GTTN are good fluorescent nanoprobes with different sub-cellular locations.

We designed and engineered this new material through changing the synthesis route, in which sodium sulphite (for GTTN) and hydrazine hydrate (for A-GQDs) were replaced with sodium thiosulfate as the reductant. This design greatly reduces the cytotoxicity in vitro (compared with A-GQDs) and allows for crossing the membrane into the cell (compared with GTTN). The results show that all the GQDs have similar sizes and shapes, and the surface charge and functional groups of S-GQDs and GTTN are also similar. So, these factors are not the cul- 
prit responsible for their different cell behaviours. According to Fig. 4g, obvious particle aggregation of SGQDs can be seen, and the particle size increases to larger than $30 \mathrm{~nm}$ from an average size of $3 \mathrm{~nm}$ (Fig. S5). As shown in Fig. S5e, obvious sediment is observed as the settling time increases, in accordance with the enlarged particle size shown in Fig. S5a-d. As shown in Fig. 4h and Fig. S5g, the ABS peak value of S-GQDs in the upper declines, while that in the bottom increases, revealing the occurrence of sedimentation as the settling time increases. It is also found that the PL intensity (Fig. 4i and Fig. S5f) and zeta potential slightly reduce in AS, which do not happen in the culture medium (Fig. S2d). As reported in our previous work, GTTN is very stable both in vitro and in vivo, due to no interaction with the proteins, and maintains its physical and chemical nature. This stability is one of the key points of GTTN targeting to tumor cell nuclei in vivo, whereas it may also be responsible for its difficulty entering cells in normal culture conditions. All the above results illustrate that the SGQDs are not very stable compared with GTTN (Fig. 4j-1 and Fig. S6) which will maintain its stability reflected in stable particle size, UV absorption and PL intensity. The aggregation state of S-GQDs is the key factor to enter the cells and distribute in lysosome [66]. Furthermore, for in vivo distribution, S-GQDs mainly distributed in liver and kidney of mice after tail vein injection of $100 \mathrm{mg} \mathrm{kg}^{-1} \mathrm{~S}$ GQDs (Fig. S7a). And it was cleaned through renal excretion as shown in Fig. S7c, but still remained in the liver after $24 \mathrm{~h}$ administration. FT-IR spectra of fresh S-GQDs, S-GQDs \& urine and S-GQDs excreted from urine show a significant difference, revealing that the S-GQDs are not like the GTTN which is stable in vivo (Fig. S7b) [50]. For in vivo toxicity, the six parameters of serum biochemistry increased gradually after $2 \mathrm{~h}$ injection and all the indicators recovered to the normal ranges after $24 \mathrm{~h}$ injection (Fig. S8), indicating that there was no obvious damage to the kidney and liver functions after clearance of S-GQDs [67].

In conclusion, this work presented a guidingsignificance example of designing carbon-based fluorescent nanoprobes to tune their physicochemical properties and biological behaviours like toxicity and cellular distribution. The obtained S-GQDs possess a series of attractive fluorescent and chemical properties such as strong absorption bands, relatively high fluorescence QY, less $\mathrm{pH}$ dependent surface charge in AS or medium, which provides it broad applications as fluorescent probes. This simple method of engineering carbon-based fluorescent nanoprobes could reduce cytotoxicity and adjust cellular distribution through regulating their physicochemical properties. By this way, we could design the bio-probes according to biological needs and expand their biological applications.

Received 15 April 2019; accepted 3 June 2019;

published online 3 July 2019

1 Schroeder KL, Goreham RV, Nann T. Graphene quantum dots for theranostics and bioimaging. Pharm Res, 2016, 33: 2337-2357

2 Kumar V, Singh V, Umrao S, et al. Facile, rapid and upscaled synthesis of green luminescent functional graphene quantum dots for bioimaging. RSC Adv, 2014, 4: 21101

3 Du Y, Guo S. Chemically doped fluorescent carbon and graphene quantum dots for bioimaging, sensor, catalytic and photoelectronic applications. Nanoscale, 2016, 8: 2532-2543

4 Zhu S, Zhang J, Qiao C, et al. Strongly green-photoluminescent graphene quantum dots for bioimaging applications. Chem Commun, 2011, 47: 6858-6860

5 Wang L, Li W, Wu B, et al. Facile synthesis of fluorescent graphene quantum dots from coffee grounds for bioimaging and sensing. Chem Eng J, 2016, 300: 75-82

6 Zeng X, Bao J, Han M, et al. Quantum dots sensitized titanium dioxide decorated reduced graphene oxide for visible light excited photoelectrochemical biosensing at a low potential. Biosens Bioelectron, 2014, 54: 331-338

7 Chen J, Zhao M, Li Y, et al. Synthesis of reduced graphene oxide intercalated $\mathrm{ZnO}$ quantum dots nanoballs for selective biosensing detection. Appl Surf Sci, 2016, 376: 133-137

8 Liu Z, Robinson JT, Tabakman SM, et al. Carbon materials for drug delivery \& cancer therapy. Mater Today, 2011, 14: 316-323

9 Tang J, Kong B, Wu H, et al. Carbon nanodots featuring efficient FRET for real-time monitoring of drug delivery and two-photon imaging. Adv Mater, 2013, 25: 6569-6574

10 Hola K, Zhang Y, Wang Y, et al. Carbon dots-Emerging light emitters for bioimaging, cancer therapy and optoelectronics. Nano Today, 2014, 9: 590-603

11 Peng Z, Han X, Li S, et al. Carbon dots: Biomacromolecule interaction, bioimaging and nanomedicine. Coord Chem Rev, 2017, 343: $256-277$

12 Wang H, Chen Q, Zhou S. Carbon-based hybrid nanogels: A synergistic nanoplatform for combined biosensing, bioimaging, and responsive drug delivery. Chem Soc Rev, 2018, 47: 4198-4232

13 Zhang Q, Jie J, Diao S, et al. Solution-processed graphene quantum dot deep-UV photodetectors. ACS Nano, 2015, 9: 1561-1570

14 Dong Y, Dai R, Dong T, et al. Photoluminescence, chemiluminescence and anodic electrochemiluminescence of hydrazidemodified graphene quantum dots. Nanoscale, 2014, 6: 1124011245

15 Chiang CW, Haider G, Tan WC, et al. Highly stretchable and sensitive photodetectors based on hybrid graphene and graphene quantum dots. ACS Appl Mater Interfaces, 2016, 8: 466-471

16 Yan J, Ye Q, Wang X, et al. CdS/CdSe quantum dot co-sensitized graphene nanocomposites via polymer brush templated synthesis for potential photovoltaic applications. Nanoscale, 2012, 4: 21092116

17 Ma WL, Li SS. Electrically controllable energy gaps in graphene quantum dots. Appl Phys Lett, 2012, 100: 163109

18 Fan Z, Li S, Yuan F, et al. Fluorescent graphene quantum dots for 
biosensing and bioimaging. RSC Adv, 2015, 5: 19773-19789

19 Markovic ZM, Ristic BZ, Arsikin KM, et al. Graphene quantum dots as autophagy-inducing photodynamic agents. Biomaterials, 2012, 33: 7084-7092

20 Amjadi M, Manzoori JL, Hallaj T. Chemiluminescence of graphene quantum dots and its application to the determination of uric acid. J Lumin, 2014, 153: 73-78

21 Kanodarwala FK, Wang F, Reece PJ, et al. Deposition of CdSe quantum dots on graphene sheets. J Lumin, 2014, 146: 46-52

22 Antonova IV, Nebogatikova NA, Prinz VY. Fluorinated graphene films with graphene quantum dots for electronic applications. J Appl Phys, 2016, 119: 224302

23 Lu L, Zhu Y, Shi C, et al. Large-scale synthesis of defect-selective graphene quantum dots by ultrasonic-assisted liquid-phase exfoliation. Carbon, 2016, 109: 373-383

24 Chen Q, Shi C, Zhang C, et al. Magnetic enhancement of photoluminescence from blue-luminescent graphene quantum dots. Appl Phys Lett, 2016, 108: 061904

25 Jiang SD, Tang G, Ma YF, et al. Synthesis of nitrogen-doped graphene-ZnS quantum dots composites with highly efficient visible light photodegradation. Mater Chem Phys, 2015, 151: 34-42

26 Zhang RX, Cai P, Zhang T, et al. Polymer-lipid hybrid nanoparticles synchronize pharmacokinetics of co-encapsulated doxorubicin-mitomycin $\mathrm{C}$ and enable their spatiotemporal co-delivery and local bioavailability in breast tumor. NanoMed-Nanotechnol Biol Med, 2016, 12: 1279-1290

27 Sun L, Zhou X, Zhang Y, et al. Enhanced field emission of graphene-ZnO quantum dots hybrid structure. J Alloys Compd, 2015, 632: 604-608

28 Pham CV, Madsuha AF, Nguyen TV, et al. Graphene-quantum dot hybrid materials on the road to optoelectronic applications. Synth Met, 2016, 219: 33-43

29 Li Y, Hu Y, Zhao Y, et al. An electrochemical avenue to greenluminescent graphene quantum dots as potential electron-acceptors for photovoltaics. Adv Mater, 2011, 23: 776-780

30 Mombrú D, Romero M, Faccio R, et al. Tuning electrical transport mechanism of polyaniline-graphene oxide quantum dots nanocomposites for potential electronic device applications. J Phys Chem C, 2016, 120: 25117-25123

31 Liang L, Kong Z, Kang Z, et al. Theoretical evaluation on potential cytotoxicity of graphene quantum dots. ACS Biomater Sci Eng, 2016, 2: 1983-1991

32 Jin Z, Owour P, Lei S, et al. Graphene, graphene quantum dots and their applications in optoelectronics. Curr Opin Colloid Interface Sci, 2015, 20: 439-453

33 Lim CS, Hola K, Ambrosi A, et al. Graphene and carbon quantum dots electrochemistry. Electrochem Commun, 2015, 52: 75-79

34 Dong Y, Tian W, Ren S, et al. Graphene quantum dots/L-cysteine coreactant electrochemiluminescence system and its application in sensing lead(II) ions. ACS Appl Mater Interfaces, 2014, 6: 16461651

35 Justin R, Tao K, Román S, et al. Photoluminescent and superparamagnetic reduced graphene oxide-iron oxide quantum dots for dual-modality imaging, drug delivery and photothermal therapy. Carbon, 2016, 97: 54-70

36 Wang L, Zhu SJ, Wang HY, et al. Common origin of green luminescence in carbon nanodots and graphene quantum dots. ACS Nano, 2014, 8: 2541-2547

37 Roy P, Periasamy AP, Chuang C, et al. Plant leaf-derived graphene quantum dots and applications for white LEDs. New J Chem, 2014,
38: 4946-4951

38 Yan L, Zhang Y, Zhang X, et al. Single layer graphene electrodes for quantum dot-light emitting diodes. Nanotechnology, 2015, 26: 135201

39 Zhu G, Xu T, Lv T, et al. Graphene-incorporated nanocrystalline $\mathrm{TiO}_{2}$ films for CdS quantum dot-sensitized solar cells. J Electroanal Chem, 2011, 650: 248-251

40 Zhu Z, Ma J, Wang Z, et al. Efficiency enhancement of perovskite solar cells through fast electron extraction: The role of graphene quantum dots. J Am Chem Soc, 2014, 136: 3760-3763

41 Zhuo S, Shao M, Lee ST. Upconversion and downconversion fluorescent graphene quantum dots: Ultrasonic preparation and photocatalysis. ACS Nano, 2012, 6: 1059-1064

42 Li Y, Chopra N. Fabrication of nanoscale heterostructures comprised of graphene-encapsulated gold nanoparticles and semiconducting quantum dots for photocatalysis. Phys Chem Chem Phys, 2015, 17: 12881-12893

43 Tetsuka H, Nagoya A, Asahi R. Highly luminescent flexible aminofunctionalized graphene quantum dots@cellulose nanofiber-clay hybrids for white-light emitting diodes. J Mater Chem C, 2015, 3: 3536-3541

44 Wang L, Wang Y, Xu T, et al. Gram-scale synthesis of singlecrystalline graphene quantum dots with superior optical properties. Nat Commun, 2014, 5: 5357

45 Zhu S, Song Y, Zhao X, et al. The photoluminescence mechanism in carbon dots (graphene quantum dots, carbon nanodots, and polymer dots): Current state and future perspective. Nano Res, 2015, 8: 355-381

46 Zhou L, Geng J, Liu B. Graphene quantum dots from polycyclic aromatic hydrocarbon for bioimaging and sensing of $\mathrm{Fe}^{3+}$ and hydrogen peroxide. Part Part Syst Charact, 2013, 30: 1086-1092

$47 \mathrm{Wu}$ X, Tian F, Wang W, et al. Fabrication of highly fluorescent graphene quantum dots using L-glutamic acid for in vitro/in vivo imaging and sensing. J Mater Chem C, 2013, 1: 4676-4684

48 Song Y, Zhu S, Zhang S, et al. Investigation from chemical structure to photoluminescent mechanism: A type of carbon dots from the pyrolysis of citric acid and an amine. J Mater Chem C, 2015, 3: 5976-5984

49 Shi F, Zhang Y, Na W, et al. Graphene quantum dots as selective fluorescence sensor for the detection of ascorbic acid and acid phosphatase via $\mathrm{Cr}(\mathrm{VI}) / \mathrm{Cr}(\mathrm{III})$-modulated redox reaction. J Mater Chem B, 2016, 4: 3278-3285

50 Lei Z, Ding L, Yao C, et al. A highly efficient tumor-targeting nanoprobe with a novel cell membrane permeability mechanism. Adv Mater, 2019, 31: 1807456

51 Pan D, Guo L, Zhang J, et al. Cutting sp2 clusters in graphene sheets into colloidal graphene quantum dots with strong green fluorescence. J Mater Chem, 2012, 22: 3314-3318

52 Pan D, Zhang J, Li Z, et al. Hydrothermal route for cutting graphene sheets into blue-luminescent graphene quantum dots. Adv Mater, 2010, 22: 734-738

$53 \mathrm{Xu} \mathrm{H}$, Zhou S, Xiao L, et al. Fabrication of a nitrogen-doped graphene quantum dot from MOF-derived porous carbon and its application for highly selective fluorescence detection of $\mathrm{Fe}^{3+}$. J Mater Chem C, 2015, 3: 291-297

54 Zhu S, Zhang J, Tang S, et al. Surface chemistry routes to modulate the photoluminescence of graphene quantum dots: From fluorescence mechanism to up-conversion bioimaging applications. Adv Funct Mater, 2012, 22: 4732-4740

55 Kim S, Hwang SW, Kim MK, et al. Anomalous behaviors of visible 
luminescence from graphene quantum dots: Interplay between size and shape. ACS Nano, 2012, 6: 8203-8208

56 He P, Sun J, Tian S, et al. Processable aqueous dispersions of graphene stabilized by graphene quantum dots. Chem Mater, 2014, 27: $218-226$

57 Canton I, Battaglia G. Endocytosis at the nanoscale. Chem Soc Rev, 2012, 41: 2718-2739

58 Liu H, Sun Y, Li Z, et al. Lysosome-targeted carbon dots for ratiometric imaging of formaldehyde in living cells. Nanoscale, 2019, 11: $8458-8463$

59 Stern ST, Adiseshaiah PP, Crist RM. Autophagy and lysosomal dysfunction as emerging mechanisms of nanomaterial toxicity. Part Fibre Toxicol, 2012, 9: 20

60 Lalwani G, D'Agati M, Khan AM, et al. Toxicology of graphenebased nanomaterials. Adv Drug Deliver Rev, 2016, 105: 109-144

61 Singh H, Sreedharan S, Tiwari K, et al. Two photon excitable graphene quantum dots for structured illumination microscopy and imaging applications: Lysosome specificity and tissue-dependent imaging. Chem Commun, 2019, 55: 521-524

62 Khlebtsov N, Dykman L. Biodistribution and toxicity of engineered gold nanoparticles: A review of in vitro and in vivo studies. Chem Soc Rev, 2011, 40: 1647-1671

63 Rejman J, Nazarenus M, Jimenez de Aberasturi D, et al. Some thoughts about the intracellular location of nanoparticles and the resulting consequences. J Colloid Interface Sci, 2016, 482: 260-266

64 He B, Shi Y, Liang Y, et al. Single-walled carbon-nanohorns improve biocompatibility over nanotubes by triggering less proteininitiated pyroptosis and apoptosis in macrophages. Nat Commun, 2018, 9: 2393

65 Zhang QQ, Yang T, Li RS, et al. A functional preservation strategy for the production of highly photoluminescent emerald carbon dots for lysosome targeting and lysosomal $\mathrm{pH}$ imaging. Nanoscale, 2018, 10: 14705-14711

66 Fiandra L, Mazzucchelli S, De Palma C, et al. Assessing the in vivo targeting efficiency of multifunctional nanoconstructs bearing antibody-derived ligands. ACS Nano, 2013, 7: 6092-6102

67 Palevsky PM. Unresolved issues in dialysis: dialysis modality and dosing strategy in acute renal failure. Seminars Dialysis, 2006, 19: $165-170$

Acknowledgements This work has been supported by the National Natural Science Foundation of China $(21371115,11025526,1175107$, 21101104 and 11422542), Shanghai University-Universal Medical Imaging Diagnostic Research Foundation (19H00100) and the Program for Changiiang Scholars and Innovative Research Team in University (IRT13078).

Author contributions Zhang $\mathrm{K}$, Yao $\mathrm{C}$ and Gao $\mathrm{W}$ designed and engineered the materials; Li C, Ding $\mathrm{L}$ and Huang $\mathrm{Y}$ conceived and performed the biological experiments and characterization of materials; Zhang $\mathrm{J}$ contributed to the discussion and paper writing. Wang $\mathrm{Y}$ convinced the idea. Wang $\mathrm{Y}$ and $\mathrm{Wu} \mathrm{M}$ wrote the paper. All authors contributed to the general discussion.

Conflict of interest The authors declare that they have no conflict of interest.

Supplementary information Experimental details and supporting data are available in the online version of the paper.

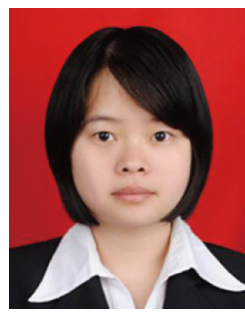

Chenchen $\mathbf{L i}$ was born in 1991. She is a $\mathrm{PhD}$ student in Prof. Minghong Wu's group and supervised by Prof. Yanli Wang. She joined Prof. Wu's group as a $\mathrm{PhD}$ student in 2016. Her research interests focus on the biosecurity and biobehavior of nanomaterials.

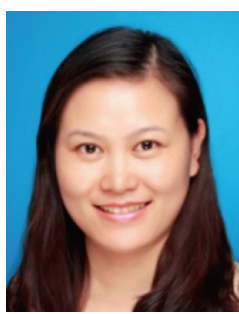

Yanli Wang obtained her $\mathrm{PhD}$ degree in environmental engineering from Shanghai University in 2010. Now she is the director of the Tumor Precision Targeting Research Center, Shanghai University, and a professor of the School of Environmental and Chemical Engineering, Shanghai University. Her main research interests include: 1 . the application of intelligent targeted fluorescent nanomaterials in tumor diagnosis; 2 . intelligent targeted nanodrug design and its application in tumor therapy; 3. the development of tumor marker detection kit; 4. biosecurity of nanomaterials.

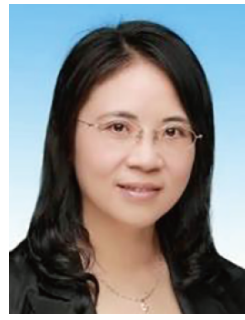

Minghong Wu obtained her $\mathrm{PhD}$ degree from Shanghai Institute of Applied Physics of Chinese Academy of Sciences in 1999. She is the vice president of Shanghai University. She is the National Outstanding Youth, Yangtze River scholar of China and the foreign academicians of Russian Academy of Engineering and Russian Academy of Science. Her research interests mainly focus on bio-effects and safety evaluation of nanomaterials and environmental pollution analysis and control.

\section{通过碳基苂光纳米探针的设计调控其亚细胞定位 和生物成像} 李晨晨 ${ }^{1,2 \dagger}$, 章康康 ${ }^{1 \dagger}$, 姚晨婕, ${ }^{1,4}$, 琳 $^{1,5}$, 黄雅男 ${ }^{1,2}$, , 尹雪莲 ${ }^{1,2}$,
章俊峰 $^{1,2}$, 高文超 ${ }^{1,2}$, 张建 ${ }^{3}$, 吴明红 ${ }^{*}$, 王艳丽 ${ }^{1,2^{*}}$

摘要 碳基荧光纳米探针在生物成像领域展现出诱人的应用前景. 本文通过调节合成方法合成了一种磺酸基修饰的石墨烯量子点(SGQDs)荧光探针. 该探针呈现出优异的光学和理化性能, 如苂光强 度高、 $\mathrm{pH}$ 稳定、表面带负电等. 研究表明其发光机理主要依赖苂 光分子发光机制. 与我们之前报道的氨基化量子点A-GQDs和肿瘤 细胞核靶向探针GTTN对比，该探针具有良好的生物安全性，可以 在相当短的时间内即跨膜进入细胞, 而GTTN在正常的体外培养条 件下无法进入细胞. 为此, 我们探究了产生该差异的原因. 结果表 明, S-GQDs与A-GQDs截然不同的合成原料导致了他们的毒性差 异, 而S-GQDs的不稳定性则是导致其进入细胞、与GTTN明显不 同的主要原因. 